\title{
The Renaissance of Antineutrophil Cytoplasmic Antibodies as a Predictor of Relapse: Ippon for Japan
}

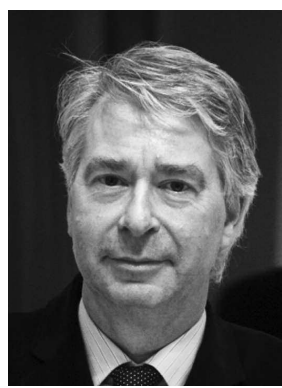

In 1985, van der Woude, et al described antineutrophil cytoplasmic antibodies (ANCA) in granulomatosis and polyangiitis (GPA, formerly Wegener's granulomatosis) and suggested that ANCA might be a useful tool for disease activity ${ }^{1}$. Subsequently, we started a prospective study in which ANCA was measured every month in patients with GPA with both physician and patient blinded to ANCA results. During a period of 16 months, 18 of 35 patients demonstrated a rise in ANCA levels; and in 17 of 18 patients, relapse followed ${ }^{2}$. Subsequently, we started a prospective trial, where patients were randomized after an ANCA rise to receive either preemptive therapy using a 9-month course of cyclophosphamide and 3 months of corticosteroids or no therapy until clinical relapse occurred. During a period of 24 months, 20 of 58 patients had ANCA rises. Nine patients were randomized for treatment and none relapsed, whereas 9 out of 11 patients were randomized for no therapy and developed a relapse. Importantly, patients receiving no treatment at the time of ANCA rise ultimately received more cyclophosphamide and prednisolone than patients on preemptive treatment ${ }^{3}$. Later, in an open-label study, the group of Niles, et al confirmed that preemptive increases in immunosuppression following ANCA rises reduced the risk of relapse ${ }^{4}$.

During the next decades, several observational studies without intervention were performed. From these studies, it was concluded that the value of repeated ANCA measurements among patients with ANCA associated vasculitis (AAV) is limited and it became more or less unethical to treat AAV patients based on ANCA levels ${ }^{5}$.

In the current issue of The Journal, Yamaguchi, et al report their findings on the occurrence of ANCA rises and preemptive increases in immunosuppression following ANCA rises in their Japanese cohort of patients with AAV. In this study, $118 \mathrm{AAV}$ patients in remission were followed during a median observation period of 41 months. In the course of the study, 57 of 118 patients (48\%) experienced an ANCA rise. Twenty-two patients received preemptive therapy, and only 1 of these patients relapsed. In contrast, relapses occurred in 29 of $35(83 \%)$ patients who were not treated immediately after an ANCA rise ${ }^{6}$.

\section{Factors Affecting Study Results}

A genetic contribution in AAV has been extensively studied ${ }^{7}$. A single nucleotide polymorphism in the HLA-DPB region on chromosome 6 (HLA-DPB $1 * 0401$ ) was found in a large percentage of patients with proteinase 3 ANCA (PR3-ANCA) associated vasculitis but not in patients with myeloperoxidase ANCA (MPO-ANCA) associated vasculitis ${ }^{7,8}$. HLA-DPB1*0401 polymorphism is rare in Japan $(4.5 \%)$ as compared to white healthy individuals (around $40 \%)^{9}$. In line with this finding, PR3-ANCA associated vasculitis is rare in Japan ( $2 \%$ of the patients in the study by Yamaguchi, et $a l)^{6}$. It has been proposed that MPO-ANCA is a better predictor for relapses than PR3-ANCA ${ }^{5}$. However, in our recent study ( $\mathrm{n}=166$ patients) we found that the predictive value of a MPO-ANCA rise was not different from the predictive value of a PR3-ANCA rise ${ }^{10}$. ANCA patterns, however, may be relevant for prediction of relapse: the reappearance of ANCA, i.e., becoming positive again after 1 or more negative results, is more predictive than ANCA rises in patients that test persistently ANCA positive during followup ${ }^{10}$.

Another relevant factor is the extent of disease. In the studies performed in the $1980 \mathrm{~s}^{2,3}$, virtually all patients with AAV included in studies had renal involvement. Also, in the current study by Yamaguchi, et al, all patients had AAV with renal involvement ${ }^{6}$. Controversy on the role of ANCA as a predictor of relapse started when studies were performed with patients with more limited AAV, i.e., without renal involvement ${ }^{11}$. Indeed, we demonstrated recently that ANCA rises correlated with relapse in patients who presented with renal disease, but not in patients who presented with non-renal disease ${ }^{10}$. These findings were recently confirmed in the samples of the RAVE study as presented recently during the 17th ANCA workshop in London ${ }^{12}$.

See MPO-ANCA level and relapse, page 1853

Personal non-commercial use only. The Journal of Rheumatology Copyright $@$ C 2015. All rights reserved. 
The therapy used to treat ANCA associated vasculitis is also relevant. In the study by Yamaguchi, et al many patients were treated with corticosteroids only ${ }^{6,13}$. We recently found that ANCA levels predicted relapses better in patients previously treated with a regimen lacking cyclophosphamide or rituximab compared to those treated with an induction regimen containing either one of these drugs ${ }^{14}$.

Another issue is the frequency of sampling. As in the studies in the $1980 \mathrm{~s}^{2,3}$, Yamaguchi, et al sampled all patients every month. Although monthly sampling may be impractical, it is now clear that frequent sampling increases the value of ANCA to predict a relapse ${ }^{10}$.

Other methodological issues are also relevant. ANCA are traditionally detected by an indirect immunofluorescence (IIF) technique ${ }^{1,2,3,15}$. For quantification of ANCA levels, however, antigen-specific tests are preferred ${ }^{15}$. Indeed, we demonstrated that ANCA rises as measured by antigen-specific ANCA tests are more strongly related to relapse compared to ANCA rises as detected by $\mathrm{IIF}^{10,16}$. Nowadays, different antigen-specific tests are used: direct ELISA tests (first generation), capture ELISA tests (second generation), and anchor ELISA tests (third generation) ${ }^{15}$. Yamaguchi, et al used ANCA tests of the first generation ${ }^{6}$. Interestingly, the association between ANCA and relapse seems stronger when newer-generation ANCA methods are used ${ }^{15}$.

Finally, definitions of ANCA rises may influence the power of an ANCA rise to predict relapse. ANCA rises are generally defined using a receiver operating characteristic (ROC) curve ${ }^{16}$. More recently, we introduced a new approach using a ROC-determined slope cutoff approach, which is even more accurate to define rises in $\mathrm{ANCA}^{10}$. Unfortunately, in the study by Yamaguchi, et al ANCA rises were poorly defined, as was accurately acknowledged as a major limitation of their study ${ }^{6}$.

\section{Controversies Not Yet Solved}

In the study by Yamaguchi, et al physicians were not blinded to ANCA results ${ }^{6}$. Knowledge of ANCA status may have been a serious bias when disease activity was assessed.

The combination of corticosteroids with either cyclophosphamide or rituximab is at present considered the standard induction regimen for patients with ANCA associated vasculitis with renal involvement ${ }^{7}$. In the study by Yamaguchi, et al, the majority of patients received only corticosteroids, whereas only a small minority of patients received cyclophosphamide, and no patient received rituximab. Moreover, a mortality rate of $23 \%$ was found whereas another $14 \%$ of patients became dialysis dependent (i.e., a non-death censored renal survival of $63 \%)^{6}$. When an induction regimen with cyclophosphamide and corticosteroids is used, we found in the last decade a non-death censored renal survival of $>75 \%{ }^{17}$. Further, in the study by Yamaguchi, et al a 5-year cumulative probability to relapse of $40 \%$ was found, which is higher than expected for
MPO-ANCA patients, i.e., $15-30 \%{ }^{7}$. These outcome data are possibly related to use of glucocorticoid monotherapy without the use of other immunosuppressives.

\section{What Future Studies Are Needed?}

Although cyclophosphamide was found to be effective as preemptive therapy ${ }^{3}$, overtreatment with this drug with its major toxicities is not acceptable. So, the preemptive use of cyclophosphamide has not been widely accepted, and other drugs such as rituximab should be considered ${ }^{18}$. Whether individualized readministration of rituximab guided by B-cell reconstitution and ANCA levels is equivalent to rituximab dosing at fixed intervals is a question currently addressed in an ongoing randomized controlled trial (MAINRITSAN2 ClinicalTrials.gov ID: NCT01731561).

Also, corticosteroid monotherapy is an option to study as preemptive therapy ${ }^{6}$. The question is: Could increased doses of corticosteroid prevent relapses after (MPO-) ANCA reappearance? MPO-ANCA patients who became negative for ANCA and subsequently retested positive for MPO-ANCA had a 40-fold higher risk to develop a relapse after the ANCA rise versus before the ANCA rise ${ }^{10}$, suggesting this might be the preferred scenario to perform a study using steroids as preemptive therapy.

Finally, more research is needed to determine why $20-50 \%$ of ANCA patients do not develop a relapse after ANCA rise. Is this because current ANCA tests are not specific for pathogenic epitopes? Or is pathogenicity determined by other qualities of ANCA such as avidity and/or glycosylation and/or sialylation ratios ${ }^{15}$ ? Otherwise, a second "hit" may be required to develop a relapse after an ANCA rise. Indeed, in a mouse model of MPO-ANCA we demonstrated the importance of such a second hit ${ }^{19}$. Whether a second hit such as infection is operative in the human situation, is, however, uncertain and should be further studied $^{14}$.

In conclusion, the report by Yamaguchi, et al revitalizes the question whether ANCA levels can be used to guide therapy in AAV. Until recently the answer was "no;" but this new study reopens the discussion and at present the answer is "maybe." As such, it is ippon ("one full point") for Japan. What is also clear, however, is that more studies are needed before such a strategy can be adopted.

JAN WILLEM COHEN TERVAERT, $M D, P h D$, Clinical and Experimental Immunology,

Maastricht University,

Maastricht, The Netherlands

Address correspondence to Prof. Cohen Tervaert, Clinical and Experimental Immunology, Maastricht University, Universiteitssingel 40, 6229ER Maastricht, the Netherlands.

E-mail: jw.cohentervaert@maastrichtuniversity.nl

\section{REFERENCES}

1. van der Woude FJ, Rasmussen N, Lobatto S, Wiik A, Permin H, van Es LA, et al. Autoantibodies against neutrophils and

Personal non-commercial use only. The Journal of Rheumatology Copyright @ 2015 . All rights reserved. 
monocytes: tool for diagnosis and marker of disease activity in Wegener's granulomatosis. Lancet 1985;1:425-9.

2. Tervaert JW, van der Woude FJ, Fauci AS, Ambrus JL, Velosa J, Keane WF, et al. Association between active Wegener's granulomatosis and anticytoplasmic antibodies. Arch Intern Med 1989;149:2461-5.

3. Tervaert JW, Huitema MG, Hené RJ, Sluiter WJ, The TH, van der Hem GK, et al. Prevention of relapses in Wegener's granulomatosis by treatment based on antineutrophil cytoplasmic antibody titre. Lancet 1990;336:709-11.

4. Han WK, Choi HK, Roth RM, McCluskey RT, Niles JL. Serial ANCA titers: useful tool for prevention of relapses in ANCA-associated vasculitis. Kidney Int 2003;63:1079-85.

5. Tomasson G, Grayson PC, Mahr AD, Lavalley M, Merkel PA. Value of ANCA measurements during remission to predict a relapse of ANCA-associated vasculitis - a meta-analysis. Rheumatology (Oxford) 2012;51:100-9.

6. Yamaguchi M, Ando M, Kato S, Katsuno T, Kato N, Kosugi T, et al. An increase of MPO-ANCA in renal ANCA-associated vasculitis patients is associated with a risk to relapse. $\mathrm{J}$ Rheumatol 2015;42:1853-60.

7. Hilhorst M, van Paassen P, Tervaert JW; Limburg Renal Registry. Proteinase 3-ANCA vasculitis versus myeloperoxidase-ANCA vasculitis. J Am Soc Nephrol 2015 May 8. [Epub ahead of print]

8. Lyons PA, Rayner TF, Trivedi S, Holle JU, Watts RA, Jayne DR, et al. Genetically distinct subsets within ANCA-associated vasculitis. N Engl J Med 2012;367:214-23.

9. Jinam TA, Nakaoka H, Hosomichi K, Mitsunaga S, Okada H, Tanaka A, et al. HLA-DPB1*04:01 allele is associated with non-obstructive azoospermia in Japanese patients. Hum Genet 2013;132:1405-11.

10. Kemna MJ, Damoiseaux J, Austen J, Winkens B, Peters J, van Paassen P, et al. ANCA as a predictor of relapse: useful in patients with renal involvement but not in patients with nonrenal disease. J Am Soc Nephrol 2015;26:537-42.

11. Kerr GS, Fleisher TA, Hallahan CW, Leavitt RY, Fauci AS, Hoffman GS. Limited prognostic value of changes in antineutrophil cytoplasmic antibody titer in patients with Wegener's granulomatosis. Arthritis Rheum 1993;36:365-71.
12. Fussner LA, Hummel AM, Schroeder D, Silva F, Cartin-Ceba R, Snyder M, et al. Predictive value of a rise in PR3-ANCA for relapse after complete remission in severe ANCA-associated vasculitis. Nephron 2015;129 Suppl 2:113-4.

13. Ozaki S, Atsumi T, Hayashi T, Ishizu A, Kobayashi S, Kumagai S, et al. Severity-based treatment for Japanese patients with MPO-ANCA-associated vasculitis: the JMAAV study. Mod Rheumatol 2012;22:394-404.

14. Kemna M, Damoiseaux J, van Paassen P, Cohen Tervaert JW. Risk factors for a relapse at a rise of ANCA in vasculitis patients with renal involvement. Nephron 2015;129 Suppl 2:214.

15. Cohen Tervaert JW, Damoiseaux J. Antineutrophil cytoplasmic autoantibodies: how are they detected and what is their use for diagnosis, classification and follow-up? Clin Rev Allergy Immunol 2012;43:211-9.

16. Boomsma MM, Stegeman CA, van der Leij MJ, Oost W, Hermans J, Kallenberg CG, et al. Prediction of relapses in Wegener's granulomatosis by measurement of antineutrophil cytoplasmic antibody levels: a prospective study. Arthritis Rheum 2000; 43:2025-33.

17. Hilhorst $M$, Wilde $B$, van Paassen $P$, Winkens B, van Breda Vriesman P, Cohen Tervaert JW; Limburg Renal Registry. Improved outcome in anti-neutrophil cytoplasmic antibody (ANCA)-associated glomerulonephritis: a 30-year follow-up study. Nephrol Dial Transplant 2013;28:373-9.

18. Cartin-Ceba R, Golbin JM, Keogh KA, Peikert T, Sánchez-Menéndez M, Ytterberg SR, et al. Rituximab for remission induction and maintenance in refractory granulomatosis with polyangiitis (Wegener's): ten-year experience at a single center. Arthritis Rheum 2012;64:3770-8.

19. Huugen D, Xiao H, van Esch A, Falk RJ, Peutz-Kootstra CJ, Buurman WA, et al. Aggravation of anti-myeloperoxidase antibody-induced glomerulonephritis by bacterial lipopolysaccharide: role of tumor necrosis factor-alpha. Am J Pathol 2005;167:47-58.

J Rheumatol 2015;42:1734-6; doi:10.3899/jrheum.150879 